\title{
Theoretical analysis of the efficiency of a V2G wireless charger for Electric Vehicles
}

\author{
Alicia Triviño, Jose M. Gonzalez-Gonzalez, and Jose A. Aguado
}

\begin{abstract}
V2G (Vehicle-to-grid) technology will report important benefits for the operation and safety of the grid. In order to facilitate the expansion of the V2G technology in a future, it is recommended to offer the drivers with easy to use methods to charge and discharge their EV batteries. In this sense, wireless chargers are expected to play a relevant role in the future electrical networks as it reduces the users' intervention. The development of this kind of system is still open to improve them in terms of their operation, their compliance and their control. An important issue for the evaluation of these systems is the efficiency, which measures the power losses occurring in the system. This paper addresses a deep study about the losses in a bidirectional wireless charger. Then, it provides with a mathematical model to characterize them. This model is validated by means of experimental results conducted in a 3.7$\mathrm{kW}$ prototype.
\end{abstract}

Index Terms-V2G, wireless charge, wireless discharge, losses, efficiency, electric vehicle, inductively-coupled power system, ICPT, bidirectional.

\section{INTRODUCTION}

$\mathbf{E}$ LECTRIC vehicles (EV) represent a clear eco-friendly mobility solution. Firstly, it is able reduce $\mathrm{CO} 2$ emissions [1-3]. On the other hand, it also helps for the integration of renewable energy sources [3, 4]. Despite these potential benefits, the proliferation of electric vehicles must be carefully controlled as a big number of them stands out for a considerable aggregated load to the grid. Market-driven solutions [5] aims at prompting the charge of the vehicles in those times when it is more convenient for the grid, avoiding the periods with a high demand too [6].

In a V2G context, the vehicles operate in an active discharge mode that needs to be also controlled [6]. Specifically, when recommended, the vehicles could decide to deliver energy to the grid from their batteries. If correctly coordinated, this operation leads to important advantages for the grid.

In order to promote the controlled charge and discharge modes and to obtain important advantages, it is highly recommended to reduce the user's intervention. This can be achieved with wireless chargers for Electric Vehicles (EV) [7].

Wireless power transfer technology can be realized by different techniques. By now, the most popular one is the one based on a pair of loosely coupled coils operating under resonant conditions. This is known as Inductively Coupled Power Transfer or ICPT [7]. In this technique, one coil is placed in the pavement (named the primary side) and the other in the chassis (known as the pickup or secondary side).

A. Triviño, J. M. Gonzalez-Gonzalez and J. A. Aguado are with the Electrical Engineering Department, Universidad de Málaga, Málaga, Spain (e-mail: atc@uma.es).
The current through the primary coil induces a voltage in the secondary coil, which is used to charge the battery. The magnetic field involved in this charger is ranged in the 20 $\mathrm{kHz}-100 \mathrm{kHz}$ interval [8]. To generate this high-frequency sinusoidal current, power converters need to be included in the charger.

The non-idealities of the switches of the power converters and the parasitic resistances of the reactive components are responsible for the losses in the whole system, which degrades the charger efficiency. This paper addresses the proposal and verification of a model to characterize the efficiency of a bidirectional wireless charger. The main contribution of our work are the following ones:

- In contrast to $[9,10]$, our work is focused on a bidirectional wireless charger operating at $85 \mathrm{kHz}$ and 3.7 $\mathrm{kW}$. The change in the frequency and on the power requires the use of specific semiconductors. In particular, SiC MOSFETs are used [11, 12]. The particularities of these devices need to be considered for the model as they clearly affect in the losses of the system and in the efficiency.

- It extends the work in [13] by focusing on the efficiency and adding a comprehensive analysis of the experimental results, which is the basis for the computation of this parameter in a prototype.

The rest of the paper is structured as follows. Section II reviews some related works about the study of losses in wireless chargers basing on ICPT technology. Section III presents the ICPT wireless charger topology used in this work. The theoretical model to compute the efficiency and the losses is presented in Section IV. Section V evaluates the methods basing on the real measurements performed in a $3.7-\mathrm{kW}$ prototype. Finally, Section VI describes the main conclusions of this work.

\section{RELATED WORK}

The losses of an EV wireless charger are mainly due to the semiconductors employed in the power converters and the parasitic resistance of the real reactive elements.

Ideal semiconductors in the power converters are operated in such a way that no losses result. However, the real behavior leads to conductive and switching losses for these elements. Conductive losses are produced because of the difference of the element from an ideal switch. To understand the losses by this event, we can rely on semiconductors models, which represent the device as an equivalent circuit with parasitic components such as resistances or capacitors. Conductive 
losses occur because of the resistances. When the current flows through the resistances, losses occur. On the other hand, the capacitors prevents the semiconductors from having instantaneous commutations, which implies switching losses.

As for the reactive components, the conductive losses are due to the resistance offered by the cables on which they are built. An equivalent model for these elements adds a series resistance to the reactive component. It is known as the ESR (Equivalent Series Resistance) and its value greatly depends on the material of the reactive components. For instance, Litz wire minimizes the skin effect and, in turn, the ESR [14].

The work in [9] makes a deep theoretical study about the switching losses of a wireless charger. The impact of them depends on the fact whether they are hard or soft. A similar study but in a low-power application is proposed in [10]. The prototype under evaluation uses a different power transfer technique working at $6.78 \mathrm{MHz}$ and transferring $2 \mathrm{~W}$.

The work in [15] presents the model for the computation of the switching losses for MOSFET in a unidirectional wireless charger. However, they do not rely on the equivalent model and they employ the parameters observed in the experimental results to derive this type of loss. The use of the parasitic capacitors, as we do in the present work, eases the estimation of the losses before the prototype is built.

Our present work uses the model presented in [9] to derive the efficiency of an EV bidirectional wireless charger. It represents an extension to the study presented in [13] as it makes a deep analysis of the experimental results.

\section{ICPT WIRELESS CHARGER FOR EV}

In this work, we will focus on ICPT technology for charging and discharging the battery of an EV. The generic scheme of a unidirectional wireless charger is represented in Figure 1.

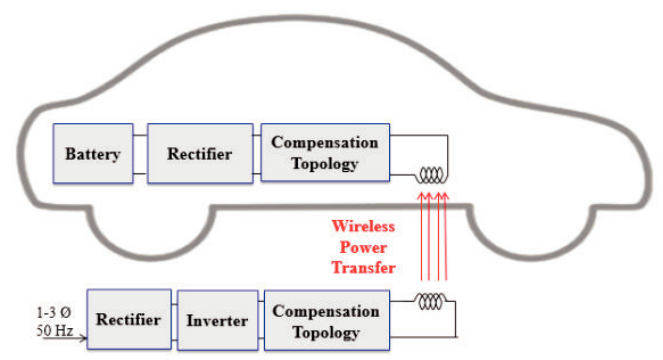

Fig. 1. Structure of a unidirectional wireless charger.

The charger is connected to the grid, whose alternating current has a frequency of $50 \mathrm{~Hz}$ or $60 \mathrm{~Hz}$ depending on the region. Nevertheless, these frequency values are not enough to transfer power inductively. Consequently, it is necessary to convert this current to high frequency current, using power electronics for that. Firstly, the alternating current is converted to direct current using a rectifier. Both single-phase and threephase rectifiers can be used. Despite its higher cost, the three phase rectifier has some advantages as for example lower losses, lower ripple factor and higher transformer utilization factor. The direct current is necessary to obtain high frequency current using an inverter. The generated high frequency current flows through the primary coil, creating a magnetic field which mainly depends on the coil structures. The magnetic field created by the primary coil induces an alternating voltage in the secondary coil, which is rectified again to provide Direct Current, which is appropriate for the battery. Additionally, a compensation system composed of capacitors is introduced on both sides to enable the operation under resonant conditions.

When implementing a bidirectional wireless charger, the power flow must be also allowed from the battery to the grid. As a consequence, the power converters should have a dual behavior to cope with the two senses of the power flows. This modification can be observed in Figure 2.

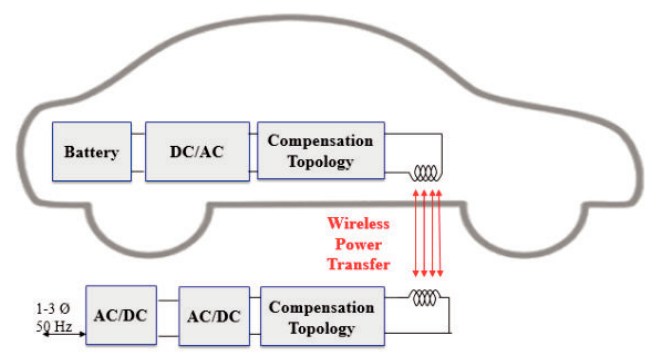

Fig. 2. Structure of a bidirectional wireless charger.

In order to allow the power flow in both senses, the primary inverter has to be capable to work also as a rectifier, while the rectifiers have to perform the functions of an inverter. This issue is solved using bidirectional AC/DC converters. As in the unidirectional wireless charger, the connection to the grid can be done through both a single-phase and three-phase converter. The three-phase converter has benefits to the singlephase converter because using three phases allows to convert the same power with less current per phase, thereby reducing the losses on the components of the converter.

\section{THEORETICAL MODEL FOR THE EFFICIENCY}

The efficiency $(\eta)$ of a wireless charger measures the relationship between the real power delivered to the load $\left(P_{\text {load }}\right)$ and the real power generated by the source $\left(P_{\text {source }}\right)$. So that:

$$
\eta=\frac{P_{\text {load }}}{P_{\text {source }}}
$$

At resonant operation and with ideal components, all the power generated by the source is delivered to the battery, that is $\eta$ equals 1 . However, due to conduction and switching losses ( $L_{\text {cond }}$ and $L_{s w}$ respectively), this parameter decreases as follows:

$$
\eta=\frac{P_{\text {source }}-L_{\text {cond }}-L_{\text {sw }}}{P_{\text {source }}}
$$

where the calculation of $L_{\text {cond }}$ and $L_{s w}$ is presented in the following subsection.

Despite having similar structures in both sides, wireless chargers can use different components in the primary and the secondary sides. One important component of the charger 


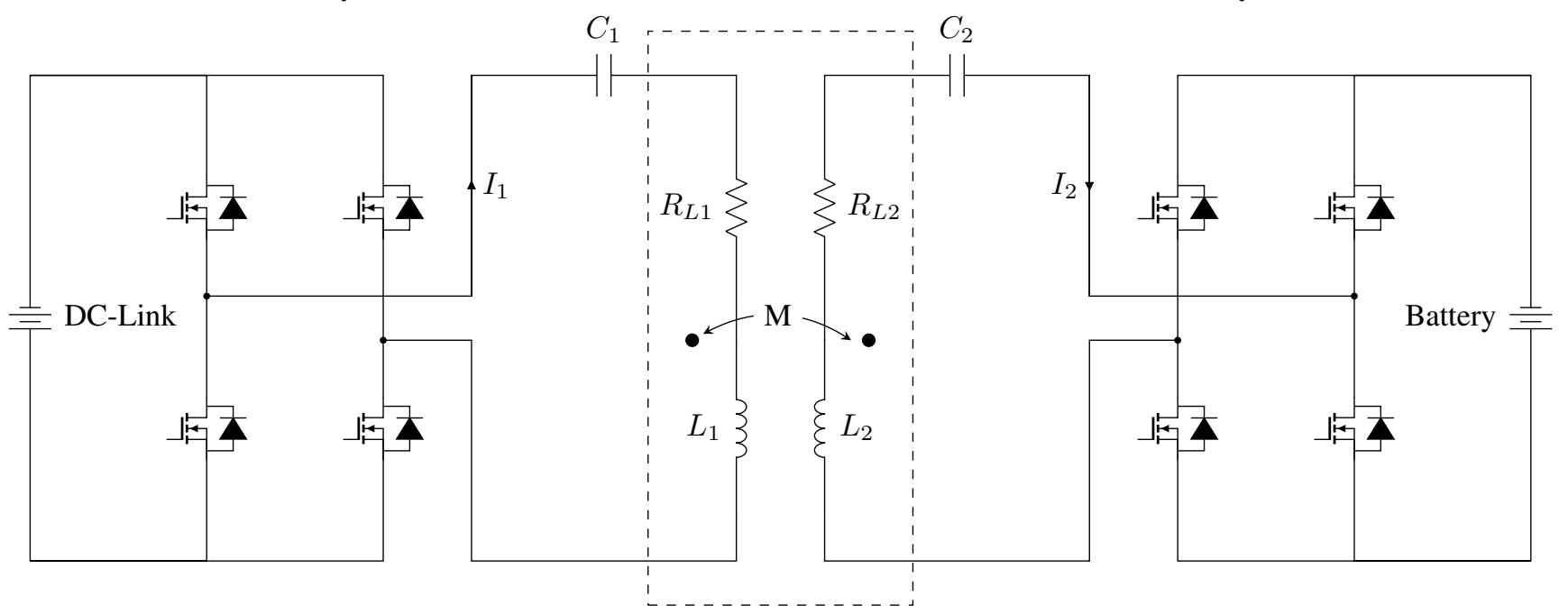

Fig. 3. Topology of the analyzed bidirectional wireless charger.

which can differ are the coils because having a bigger coil on the primary side can help with misalignments. Different coils also require different compensation systems. Due to this asymmetrical structure of the bidirectional wireless charger, it is necessary to define two efficiencies, one for each sense of the power flow. Thus, we have $\eta^{c h}$ to characterize the power flow from the grid to the battery and $\eta^{\text {dis }}$ to define the charger performance in the opposite sense. We can reformulate Eq. 2 as:

$$
\eta^{c h}=\frac{P_{g r i d}-L_{c o n d}^{c h}-L_{s w}^{c h}}{P_{g r i d}}
$$

and

$$
\eta^{d i s}=\frac{P_{g r i d}-L_{c o n d}^{d i s}-L_{s w i t c h}^{d i s}}{P_{\text {grid }}}
$$

where $P_{\text {grid }}$ represents the real power generated by the grid and $P_{b a t}$ is the real power delivered by the EV battery.

In the next subsections, we formulate the equations to estimate both efficiencies for the bidirectional wireless charger topology presented in Fig. 3.

\section{A. Charge mode}

In this operation mode, the DC/AC power converter in the primary side acts an inverter whereas the AC/DC power converter in the pickup is a rectifier. The conduction losses are:

$$
L_{c o n d}^{c h}=L_{c o n, i n v}^{c h}+L_{c o n, r e c}^{c h}+L_{c o i l s}^{c h}+L_{m a t c h}^{c h}
$$

where $L_{c o n, i n v}^{c h}$ are the conductions losses of the inverter, $L_{c o n, r e c}^{c h}$ are the conductions losses of the rectifier, $L_{c o i l s}^{c h}$ are the losses in the coils and $L_{\text {match }}^{c h}$ are the losses in the compensation system. The superscript $c h$ indicates that these values correspond to the charging mode.
In order to simplify the control, a full-bridge topology with a phase-shift control of a duty cycle equal to $50 \%$ is employed. In this scheme, there is only one transistor in each leg in conduction. Thus, the conduction losses of the inverter are:

$$
L_{c o n, i n v}^{c h}=2 \cdot R_{d s} \cdot \widehat{I}_{1}^{2}
$$

being $\widehat{I}_{1}$ the rms (root-mean squared) current in the primary side and $R_{d} s$ the internal resistance drain-source of the MOSFET in the inverter.

For the conduction losses of the rectifier, we also rely on the equivalent model of the diodes. In a full-bridge rectifier, two diodes are simultaneously conducting, both of them provoking some losses as:

$$
L_{c o n, r e c}^{c h}=2 \cdot R_{d} \cdot \widehat{I}_{r e c}^{2}+2 \cdot V_{t h} \cdot \widehat{I}_{r e c}
$$

where $R_{d}$ represents the internal resistance of the diodes, $V_{t} h$ their forward voltage and $\widehat{I}_{r e c}$ the rms value of the current traversing these elements. In a series-series compensation topology as we are using in this work, $I_{r} e c$ equals $I_{2}$, that is, the secondary current.

The coils and the matching networks also incur in conduction losses to the system due to their parasitic resistances. Specifically, the losses of the coils are estimated as:

$$
L_{\text {coils }}=R_{L 1} \cdot \widehat{I}_{1}^{2}+R_{L 2} \cdot \widehat{I}_{2}^{2}
$$

where $R_{L 1}$ and $R_{L 2}$ are the resistances associated to the primary and secondary coil respectively.

The currents injected to the coils are the same as those in the capacitors as a series-series compensation network is used. Thus, the conduction losses in the matching networks are:

$$
L_{\text {match }}=R_{C 1} \cdot \widehat{I}_{1}^{2}+R_{C 2} \cdot \widehat{I}_{2}^{2}
$$

where $R_{C 1}$ and $R_{C 2}$ are the resistances associated to the primary and secondary capacitors respectively 
Concerning the switching losses, they are due to the inverter. Theoretically, these losses can be estimated by the addition of multiple terms, being the one due to the output capacitance $\left(C_{o s s}\right)$ the most relevant $\left(L_{C o s s}\right)$. Thus, we simplify as:

$$
L_{s w}^{c h} \cong L_{C o s s}=\frac{1}{2} f_{s} C_{o s s} V_{d s}^{2}
$$

being $f_{s}$ the switching frequency and $V_{d s}$ the voltage between the drain and the source.

\section{B. Discharge mode}

In the discharge mode, the power flow from the battery to the grid. Making use of the dual behavior of the power converters, in this operation mode, the power converter attached to the battery acts as an inverter while the power converter in the primary side works as a rectifier.

In this way, the conduction losses can be expressed as:

$$
L_{\text {cond }}^{d i s}=L_{\text {con }, \text { inv }}^{d i s}+L_{\text {con,rec }}^{\text {dis }}+L_{\text {coils }}^{\text {dis }}+L_{\text {match }}^{\text {dis }}
$$

where the superscript $c h$ indicates these values correspond to the discharging mode.

Although the conduction losses of the coils $\left(L_{\text {coils }}\right)$ and the compensation system $\left(L_{\text {match }}\right)$ can be calculated following the same equations of the charging mode (Eq. 8 and 9), the conduction losses of the inverter and the rectifier differ in the current values because in this mode they are working on the secondary and the primary side respectively. Theses losses can be computed as:

$$
\begin{gathered}
L_{c o n, i n v}^{d i s}=2 \cdot R_{d s} \cdot \widehat{I}_{2}^{2} \\
L_{c o n, r e c}^{\text {dis }}=2 \cdot R_{d} \cdot \widehat{I}_{1}^{2}+2 \cdot V_{t h} \cdot \widehat{I}_{1}
\end{gathered}
$$

Finally, the switching losses are estimated following the same equation of the charging mode (Eq. 10).

\section{VALIDATION OF THE MODEL: EXPERIMENTAL RESULTS}

The developed theoretical model is verified with a $3.7-\mathrm{kW}$ bidirectional prototype based on ICPT technology. The coils are both square but with different sizes. The compensation networks are uniresonant and in series with the coils. As for the magnetic field, it is generated at $85 \mathrm{kHz}$ as recommended by SAE TIR J2954 [16]. CREE C2M0080120D SiC MOSFETs are the components of the power converters as they support the power demanded and the switching frequency. The main properties of the charger, including the values related to the non-idealities of the components, are summarized in Table I.

Firstly, we derive the theoretical results for the losses and the efficiencies considering the model presented in the previous Section. These results are exposed in Table II.

The resistance of the coils has the highest impact in the efficiency of the system. The rest of the components of the system have similar losses with the exception of the switching losses of the inverter which, thanks to the use of $\mathrm{SiC}$ MOSFETs, are negligible. Due to security reasons with the operation of the battery, the power transferred in the discharge

\begin{tabular}{|c|c|c|c|}
\hline \multicolumn{2}{|c|}{ Charger specifications } & \multicolumn{2}{|c|}{$\begin{array}{l}\text { TX-RX parameters } \\
\text { (prototype values) }\end{array}$} \\
\hline Output & $\begin{array}{l}3.7 \mathrm{~kW} \\
300 \mathrm{~V}\end{array}$ & $L_{1}[m H]$ & 240.5 \\
\hline fs $[k H z]$ & 85 & $L_{2}[m H]$ & 230.6 \\
\hline \multicolumn{2}{|c|}{ Coils geometry } & $C_{1}[n F]$ & 14.3 \\
\hline $\begin{array}{c}\text { Primary coil } \\
{\left[\mathrm{m}^{2}\right]}\end{array}$ & $0.75 \times 0.75$ & $C_{2}[n F]$ & 15.6 \\
\hline $\begin{array}{l}\text { Secondary } \\
\text { coil }\left[m^{2}\right]\end{array}$ & $0.5 \times 0.5$ & $R_{L_{1}}[m \Omega]$ & 196 \\
\hline \multicolumn{2}{|c|}{ C2M0080120D MOSFET } & $R_{L_{2}}[m \Omega]$ & 143 \\
\hline$R_{d}\lfloor m \Omega]$ & 40 & $R_{C_{1}}[m \Omega]$ & 67 \\
\hline$V_{t h}[V]$ & 0.98 & $R_{C_{2}}[m \Omega]$ & 52 \\
\hline$C_{o s s}[p F]$ & 80 & $M[m H]$ & 54.5 \\
\hline$R_{d s}[m \Omega]$ & 80 & $\begin{array}{c}k= \\
M\left(L_{1} L_{2}\right)^{1 / 2}\end{array}$ & 0.231 \\
\hline
\end{tabular}
mode has been reduced.
TABLE I

PARAMETERS OF THE ICPT SYSTEM.

TABLE II

COMPUTED LOSSES.

\begin{tabular}{|c|c|c|c|}
\hline \multicolumn{2}{|c|}{ Charge mode } & \multicolumn{2}{c|}{ Discharge mode } \\
\hline$L_{c o n, i n v}[W]$ & 25 & $L_{c o n, i n v}[W]$ & 3.3 \\
\hline$L_{\text {sw,inv }}[W]$ & 1 & $L_{\text {sw,inv }}[W]$ & 1 \\
\hline$L_{\text {con,rec }}[W]$ & 34 & $L_{\text {con,rec }}[W]$ & 15 \\
\hline$L_{\text {coils }}[W]$ & 64 & $L_{\text {coils }}[W]$ & 11 \\
\hline$L_{\text {match }}[W]$ & 23 & $L_{\text {match }}[W]$ & 4 \\
\hline
\end{tabular}

To validate the computed results, the losses are also carried out by obtaining the real power which flows for each component of the system. To get these values is necessary to analyze the oscilloscope captures both at the input and at the output of the components, with which the current and voltage are taken into account. Figure 4 shows an oscilloscope capture of the current and the voltage measure at the output of the primary DC/AC converter in charge mode. As can be observed, voltage measurement consists on a square signal which can be used to computed the real power assuming a fundamental harmonic approximation. The difference between input and output real power of each component corresponds to the losses.

The measurements of the electrical signals in the prototype leads to the values exposed in Table III.

The following subsections presents the calculation methods to obtain the results from the experimental measurements. Using these equations and the measures of Table III, the prototype losses has been computed to validate the model. These losses are shown in Table IV.

As can be observed, the use of the model based on the nonidealities leads to higher losses estimation in comparison with those derived from the analysis of the waveforms. In particular, for the charge mode, the inverter is assumed to loss $26 \mathrm{~W}$ whereas the waveform analysis states that these losses 


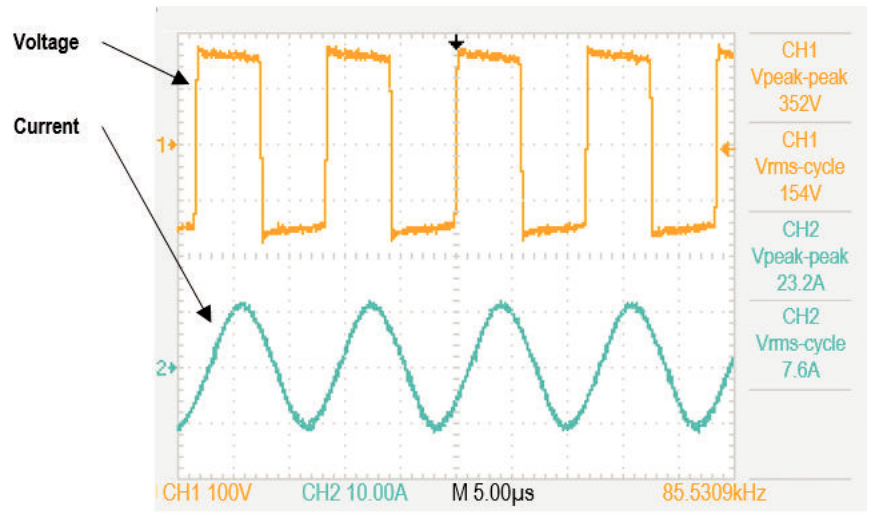

Fig. 4. Voltage (channel 1) and current (channel 2) measurements at the output of the primary DC/AC converter.

TABLE III

ELECTRICAL SIGNALS MEASURED IN THE PROTOTYPE.

\begin{tabular}{|c|c|c|}
\hline Electrical signals & Charge mode & Discharge mode \\
\hline$V_{\text {inv }}$ input $[V]$ & 288 & 298 \\
\hline$V_{\text {inv }}$ output $[V]$ & 290 & 293 \\
\hline$I_{\text {inv }}$ input $[A]$ & 12.56 & 4.56 \\
\hline$V_{\text {inv }_{\text {input }}}[A]$ & 13.78 & 5.14 \\
\hline$V_{\text {rec }_{\text {input }}}[V]$ & 285 & 247 \\
\hline$V_{\text {rec output }}[V]$ & 288 & 250 \\
\hline$I_{\text {rec }}$ input $[A]$ & 13.74 & 6.02 \\
\hline$I_{\text {rec }}$ output $[A]$ & 12.16 & 5.30 \\
\hline
\end{tabular}

are $20 \mathrm{~W}$. Concerning the rectifier operation, this difference is $11 \mathrm{~W}$. For the discharge mode, there are also some deviations. Specifically, the difference in the inverter is $1.3 \mathrm{~W}$ whereas the rectifier is associated to a deviation equals to $1 \mathrm{~W}$. Taking into account the total values, the differences are significant. This is due to the errors in the measurements, which impact on both the waveform analysis and on the model based on the nonidealities.

\section{A. Charge mode}

Concerning the output of the primary inverter, the voltage is a square-wave of $V_{\text {inv }}$ output amplitude while the current is inphase and sinusoidal (with a peak value equals to $I_{\text {inv }}{ }_{\text {output }}$ ). The shape and phase of the output current is the consequence of forcing the system to operate under resonant conditions. For the active power, we must extract the peak value of the fundamental harmonic of the voltage signal $\left(V_{\text {inv }}^{1}{ }_{\text {output }}\right)$ and operate as follows:

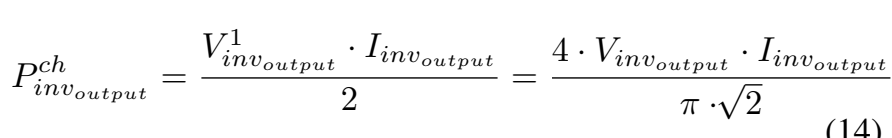

Thus, the losses in the primary inverter $\left(L_{i n v}^{c h}\right)$ are:

$$
L_{\text {inv }}^{c h}=P_{\text {inv } v_{\text {input }}}^{c h}-P_{\text {inv }}^{c h}
$$

TABLE IV

LOSSES COMPUTED FROM THE ELECTRICAL SIGNALS MEASURED IN THE PROTOTYPE.

\begin{tabular}{|c|c|c|c|}
\hline \multicolumn{2}{|c|}{ Charge mode } & \multicolumn{2}{c|}{ Discharge mode } \\
\hline$L_{\text {inv }}^{c h}[W]$ & 20 & $L_{\text {inv }}^{\text {dis }}[W]$ & 3 \\
\hline$L_{\text {coils }}+L_{\text {match }}[W]$ & 73 & $L_{\text {coils }}+L_{\text {match }}[W]$ & 17 \\
\hline$L_{\text {rec }}^{\text {ch }}[W]$ & 23 & $L_{\text {rec }}^{\text {dis }}[W]$ & 14 \\
\hline
\end{tabular}

The rectifier input consists of a square-wave voltage (with a peak value of $V_{\text {recinput }_{\text {in }}}$ ) and a sinusoidal current wave with a peak value equal to $I_{\text {recinput }}$. Applying the decomposition of harmonics, we can state that:

$$
P_{\text {rec }}^{c h \text { input }}=\frac{4 \cdot V_{r e c_{\text {input }}} \cdot I_{\text {rec }} \text { input }}{\pi \cdot \sqrt{2}}
$$

The output of the rectifier in conjunction of the low-pass filter results in two constant signals for voltage and current. The voltage equals to $V_{\text {rec output }}$ while the current is $I_{\text {rec output }}$. This lead to an output power computed as follows:

$$
P_{\text {rec output }}^{\text {ch }}=V_{\text {recoutput }} \cdot I_{\text {rec output }}
$$

Consequently, the losses in the controlled rectifier $\left(L_{r e c}^{c h}\right)$ are:

$$
L_{r e c}^{c h}=P_{r e c_{\text {input }}}^{c h}-P_{\text {rec }}^{c h}
$$

\section{B. Discharge mode}

In the discharge mode, the battery of the EV provides energy to the grid so that the power flow is reverse to the previous mode. This means that the power converter attached to the battery acts as an inverter while the power converter in the primary side works as a rectifier. The waveforms of the converters change according to their new functionality but it follows a form equivalent to the previous case. Thus, these are the losses that are defined in a different way in comparison with the afore mentioned definitions. Alternatively, $L_{c o n, i n v}$, $L_{\text {sw,inv }}, L_{\text {con,rec }}, L_{\text {coils }}$ and $L_{\text {match }}$ can be computed as previously. In the discharge mode, for the inverter attached to the battery:

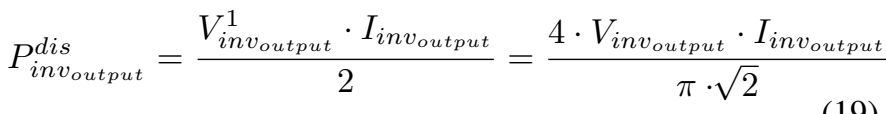

$$
\begin{aligned}
& P_{\text {inv }_{\text {input }}}^{\text {dis }}=V_{\text {inv } v_{\text {input }}} \cdot I_{\text {inv } v_{\text {input }}}
\end{aligned}
$$

On the other hand, the losses in the primary inverter $\left(L_{i n v}^{d i s}\right)$ are:

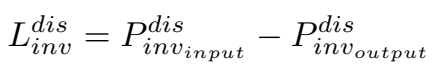

The rectifier input, which is now in the primary side, corresponds with a square-wave voltage (with a peak value of $V_{\text {recinput }}$ ) and a sinusoidal current wave with a peak value equal to $I_{r e c_{\text {input }}}$. Applying the decomposition of harmonics, we can assure that: 


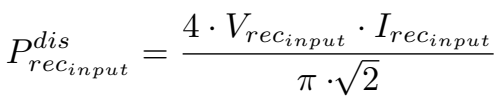

The output of the rectifier is now connected to the grid.

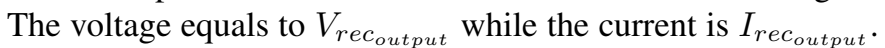
Both signals are constant as a low-pass filter is used. This implies that the output power is computed as follows:

$$
P_{\text {rec output }}^{\text {dis }}=V_{\text {recoutput }} \cdot I_{\text {rec output }}
$$

As a consequence, the losses in the controlled rectifier for the discharge mode $\left(L_{\text {rec }}^{\text {dis }}\right)$ are:

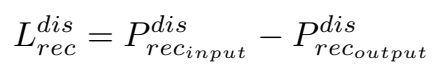

\section{CONCLUSION}

This paper presents a model for the estimation of the charge and discharge efficiency in a bidirectional ICPT wireless charger for EV. Specifically, the model relies on the equivalent circuit of the semiconductors and of the reactive components to derive the conduction and the switching losses of the system. The model is contrasted with the experimental results obtained in a $3.7 \mathrm{~kW}$ wireless charger at $85 \mathrm{kHz}$. There exist some differences among them, which are assumed to be due to measurement errors.

As future work, we intend to model the switching losses when the resonant conditions do not hold because of misalignments between the two coils.

\section{REFERENCES}

[1] L. Canals Casals, E. Martinez-Laserna, B. Amante García, and N. Nieto, "Sustainability analysis of the electric vehicle use in Europe for CO2 emissions reduction," Journal of Cleaner Production, vol. 127, pp. 425437, 2016.

[2] E. A. Moreira Falcão, A. C. Rodrigues Teixeira, and J. R. Sodré, "Analysis of CO2emissions and techno-economic feasibility of an electric commercial vehicle," Applied Energy, vol. 193, pp. 297-307, 2017.

[3] A. Triviño, M. Longo, and F. Foiadelli, "Reduction of $\mathrm{CO} 2$ emissions using RES to recharge EVs : the Spanish case," International Conference on Renewable Energies and Power Quality (ICREPQ'17), vol. 1, no. 15, 2017.

[4] K. E. Forrest, B. Tarroja, L. Zhang, B. Shaffer, and S. Samuelsen, "Charging a renewable future: The impact of electric vehicle charging intelligence on energy storage requirements to meet renewable portfolio standards," Journal of Power Sources, vol. 336, pp. 63-74, 2016.

[5] M. A. Lopez, S. De La Torre, S. Martin, and J. A. Aguado, "Demandside management in smart grid operation considering electric vehicles load shifting and vehicle-to-grid support," International Journal of Electrical Power and Energy Systems, vol. 64, pp. 689-698, 2015.

[6] K. M. Tan, V. K. Ramachandaramurthy, and J. Y. Yong, "Integration of electric vehicles in smart grid: A review on vehicle to grid technologies and optimization techniques," Renewable and Sustainable Energy Reviews, vol. 53, pp. 720-732, 2016.

[7] K. A. Kalwar, M. Aamir, and S. Mekhilef, "Inductively coupled power transfer (ICPT) for electric vehicle charging - A review," Renewable and Sustainable Energy Reviews, vol. 47, pp. 462-475, 2015.

[8] A. Triviño-Cabrera, J. Aguado, and J. González, "Analytical characterisation of magnetic field generated by ICPT wireless charger," Electronics Letters, vol. 53, no. 13, pp. 871-873, jun 2017.

[9] G. Di Capua, J. A. A. Sanchez, A. T. Cabrera, D. F. Cabrera, N. Femia, G. Petrone, and G. Spagnuolo, "A losses-based analysis for electric vehicle wireless chargers," in 2015 International Conference on Synthesis, Modeling, Analysis and Simulation Methods and Applications to Circuit Design (SMACD). IEEE, sep 2015, pp. 1-4.
[10] G. Di Capua, N. Femia, G. Petrone, G. Lisi, D. Du, and R. Subramonian, "Power and efficiency analysis of high-frequency Wireless Power Transfer Systems," International Journal of Electrical Power \& Energy Systems, vol. 84, pp. 124-134, jan 2017.

[11] N. Oswald, P. Anthony, N. McNeill, and B. H. Stark, "An Experimental Investigation of the Tradeoff between Switching Losses and EMI Generation With Hard-Switched All-Si, Si-SiC, and All-SiC Device Combinations," IEEE Transactions on Power Electronics, vol. 29, no. 5, pp. 2393-2407, may 2014.

[12] R. A. Wood and T. E. Salem, "Evaluation of a 1200-V, 800-A All-SiC Dual Module," IEEE Transactions on Power Electronics, vol. 26, no. 9, pp. 2504-2511, sep 2011.

[13] A. Trivino, J. M. Gonzalez-Gonzalez, and J. A. Aguado, "Evaluation of losses in a bidirectional wireless power transfer system for electric vehicles," in 2017 IEEE International Conference on Environment and Electrical Engineering and 2017 IEEE Industrial and Commercial Power Systems Europe (EEEIC / I\&CPS Europe). IEEE, jun 2017, pp. 1-5.

[14] Q. Deng, J. Liu, D. Czarkowski, M. K. Kazimierczuk, M. Bojarski, $\mathrm{H}$. Zhou, and W. Hu, "Frequency-Dependent Resistance of LitzWire Square Solenoid Coils and Quality Factor Optimization for Wireless Power Transfer,' IEEE Transactions on Industrial Electronics, vol. 63, no. 5, pp. 2825-2837, may 2016. [Online]. Available: http://ieeexplore.ieee.org/document/7383291/

[15] A. Moradi, F. Tahami, and M. A. GhaziMoghadam, "Wireless Power Transfer Using Selected Harmonic Resonance Mode," IEEE Transactions on Transportation Electrification, vol. 3, no. 2, pp. 508-519, 2017. [Online]. Available: http://ieeexplore.ieee.org/document/7903716/

[16] SAE International, "Wireless Power Transfer for Light-Duty PlugIn/Electric Vehicles and Alignment Methodology (SAE TIR J2954)." [Online]. Available: http://standards.sae.org/wip/j2954/ 